\title{
Ministry of Science and Technology
}

\section{Correction to: Novel mutation G324C in WNT1 mapped in a large Pakistani family with severe recessively inherited Osteogenesis Imperfecta}

Mehran Kausar ${ }^{1,2}$, Saima Siddiqi ${ }^{2}$, Muhammad Yaqoob ${ }^{3}$, Sajid Mansoor ${ }^{4,10}$, Outi Makitie ${ }^{5,6}$, Asif Mir ${ }^{7}$, Chiea Chuen Khor ${ }^{8}$, Jia Nee Foo ${ }^{8,9^{*+}}$ and Mariam Anees ${ }^{1 *+}$

\section{Correction to: J Biomed Sci}

https://doi.org/10.1186/s12929-018-0481-x

In the original publication of this article [1], there are two errors in the article which the cDNA position of the pathogenic variant WNT1 p.Gly324Cys should be c.970G $>$ T instead of c.1168G $>$ T.

\begin{abstract}
Author details
'Department of Biochemistry, Faculty of Biological Sciences,

Quaid-i-AzamUniversity, University Road, Islamabad 45320, Pakistan. ${ }^{2}$ Institute of Biomedical and Genetic Engineering (IBGE) Islamabad, Islamabad 44000, Pakistan. ${ }^{3}$ Department of Genetics, Children Hospital, Lahore, Pakistan.

${ }^{4}$ Atta-ur-Rehman School of Applied Biosciences, NUST, Islamabad, Pakistan. ${ }^{5}$ Children's Hospital, University of Helsinki and Helsinki University Hospital, Helsinki, Finland. ${ }^{6}$ Folkhälsan Institute of Genetics, Helsinki, Finland. ${ }^{7}$ Department of Bioinformatics \& Biotechnology, Faculty of Basic and Applied Sciences, International Islamic University (IIU), H-10, Islamabad 44000, Pakistan. ${ }^{8}$ Human Genetics, A*STAR, Genome Institute of Singapore, Singapore 138672, Singapore. 'Lee Kong Chian School of Medicine, Nanyang Technological University, Singapore 308232, Singapore. ${ }^{10}$ Department of Microbiology, Faculty of Life Sciences, University of Central Punjab, Lahore, Pakistan.
\end{abstract}

Received: 22 April 2019 Accepted: 22 April 2019

Published online: 28 April 2019

\section{Reference}

1. Kausar, et al. Novel mutation G324C in WNT1 mapped in a large Pakistani family with severe recessively inherited Osteogenesis Imperfecta. J Biomed Sci. 2018;25:82 https://doi.org/10.1186/s12929-018-0481-x.

\footnotetext{
*Correspondence: foojn@gis.a-star.edu.sg; jianee.foo@ntu.edu.sg; mariam@qau.edu.pk; mariamanees@yahoo.com

${ }^{\dagger}$ Jia Nee Foo and Mariam Anees contributed equally to this work.

${ }^{8}$ Human Genetics, A*STAR, Genome Institute of Singapore, Singapore 138672,

Singapore

'Department of Biochemistry, Faculty of Biological Sciences,

Quaid-i-AzamUniversity, University Road, Islamabad 45320, Pakistan

Full list of author information is available at the end of the article
}

(c) The Author(s). 2019 Open Access This article is distributed under the terms of the Creative Commons Attribution 4.0 International License (http://creativecommons.org/licenses/by/4.0/), which permits unrestricted use, distribution, and reproduction in any medium, provided you give appropriate credit to the original author(s) and the source, provide a link to the Creative Commons license, and indicate if changes were made. The Creative Commons Public Domain Dedication waiver (http://creativecommons.org/publicdomain/zero/1.0/) applies to the data made available in this article, unless otherwise stated. 